Laboratoire de l'Informatique du Parallélisme

École Normale Supérieure de Lyon

Unité Mixte de Recherche CNRS-INRIA-ENS LYON

$\mathrm{n}^{\mathrm{o}} 5668$

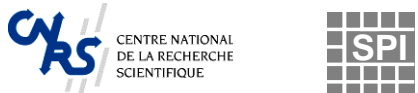

On the structure of some spaces of tilings

Eric Rémila

April 2000

École Normale Supérieure de Lyon

46 Allée d'Italie, 69364 Lyon Cedex 07, France Téléphone : $+33(0) 4.72 .72 .80 .37$

Télécopieur : +33(0)4.72.72.80.80 Adresse électronique : lip@ens-lyon.fr 


\title{
On the structure of some spaces of tilings
}

\author{
Eric Rémila
}

April 2000

\begin{abstract}
We study the structure of the set of tilings of a polygon $P$ with bars of fixed length. We obtain a undirected graph connecting two tilings if one can pass from one tile to the other one by a flip (i. e a local replacement of tiles).

Using algebraic tools (as tiling groups and their quotients and subgroups), we give a formula to compute the distance in this graph (i. e. the minimal number of necessary flips) between two tilings. Moreover, we prove that, for each pair $\left(T, T^{\prime}\right)$ of tilings, the set $\Upsilon_{T, T^{\prime}}$ formed from tilings which are in a path of minimal length from $T$ to $T^{\prime}$ canonically has a structure of distributive lattice.
\end{abstract}

Keywords: tiling, group, lattice

\section{Résumé}

Nous tudions ici l'ensemble des pavages d'un polygone $P$ par des barres de longueur fixe. Nous obtenons un graphe non orient en reliant deux pavages si l'on peut passer de l'un a l'autre par une transformation locale appele flip.

Avec des outils algbriques (les groupes de pavages et leurs quotients et sous groupes), nous donnons une formule pour calculer la distance dans ce graphe (i. e. le nombres de flips necessaires) entre deux pavages. De plus, nous prouvons que, pout toute paire $\left(T, T^{\prime}\right)$ de pavages, l'ensemble $\Upsilon_{T, T^{\prime}}$ form par les pavages qui sont sur une geodsique entre $\mathrm{T}$ et $\mathrm{T}$ ' est canonique ment muni s'une structure de teillis distributif.

Mots-clés: pavage, groupe, treillis 


\title{
On the structure of some spaces of tilings
}

\author{
Eric Rémila \\ LIP, CNRS umr 5668, ENS-Lyon \\ 46 allée d'Italie, 69364 Lyon cedex 07, France \\ and \\ GRIMA, IUT Roanne, Université Jean Monnet Saint-Etienne \\ 20 avenue de Paris, 42334 Roanne cedex, France \\ Eric.Remila@univ-st-etienne.fr
}

\begin{abstract}
We study the structure of the set of tilings of a polygon $P$ with bars of fixed length. We obtain a undirected graph connecting two tilings if one can pass from one tile to the other one by a flip (i. e a local replacement of tiles).

Using algebraic tools (as tiling groups and their quotients and subgroups), we give a formula to compute the distance in this graph (i. e. the minimal number of necessary flips) between two tilings. Moreover, we prove that, for each pair $\left(T, T^{\prime}\right)$ of tilings, the set $\Upsilon_{T, T^{\prime}}$ formed from tilings which are in a path of minimal length from $T$ to $T^{\prime}$ canonically has a structure of distributive lattice.
\end{abstract}

\section{Introduction}

In 1990, J. H. Conway and J. C. Lagarias [1] introduced the notion of tiling groups, which is a very powerful tool to study tiling problems. Using tiling groups, a lot of necessary conditions for a simply connected figure to be tileable (see [3], [4]) were discovered and unified.

This work has been prolonged by W. P. Thurston [9] who especially studied tilings formed from dominoes (i. e. rectangles $2 \times 1$ ) and tilings formed from calissons (i. e. lozenges formed from two equilateral triangles of unit side, with a common edge). For these examples, W. P. Thurston [9] introduced the notion of height function associated to a tiling of polygon $P$. Such a height function permits to encode each tiling as a mappings from vertices in $P$ to the set $Z$ of relative integers.

Using this new notion, a linear algorithm which, given a polygon as input, produces a tiling of this polygon as output (if there exists one tiling ; otherwise the claim of the impossibility is the output) is exhibited.

W. P. Thurston's ideas have been taken again by C. Kenyon and R. Kenyon [2], who obtained a tiling algorithm for the case when tilings are formed from $m$-bars (rectangles of length $m$ and unit width), and E. Rémila [5], who obtained tiling algorithms for the case when tilings are formed from equilateral triangles of side of length 2 and "leaning dominoes" (parallelograms formed from four 
equilateral triangles of sides of unit length). For these authors, height functions appear as heights on special trees.

Each of these authors also uses local transformations, called flips, which permit to transform a tiling into another one (for example, for dominoes a flip is the replacement of two tiles covering a $2 \times 2$ square by the two other tiles covering the same square). The space of tilings of $P$ is the (undirected) graph $G_{P}=\left(V_{P}, E_{P}\right)$ whose vertices are the tilings of $P$, and two tilings are linked by an edge if and only if one can pass from a tiling to the other one using only one flip. This space has been precisely studied for the cases of dominoes and calissons [8] [6]. The main result is that the space of tilings has a canonical structure of distributive lattice. At the opposite, before, the only result known about spaces of tilings introduced in [2] and [5] was the connectivity of these spaces.

In this paper, which is an extended and improved version of [7], we first study the space of tilings with $m$-bars. After recalling general notions about tiling groups and their applications (Section 2), we focus on the structure of the tiling group used for $m$-bars (Section 3 ). We especially show the importance of a special normal subgroup of the main quotient of the tiling group. Using this subgroup, we are able to give an algebraic characterization of functions which encode tilings, and give definition of a distance between tilings. We prove (Section 4) that this distance is (up to a multiplicative constant) the distance in the space of tilings, i. e. the minimal number of necessary flips to transform a tiling into another one. This fact gives the flip formula, which permits to compute this number of flips, and an algorithm to find a shortest path between two tilings given as input. From the algebraic characterization of functions which encode tilings, we prove (Section 5) for each pair $\left(T, T^{\prime}\right)$ of tilings, the subgraph of the space of tilings induced by tilings which are in a path of minimal length from $T$ to $T^{\prime}$, canonically has a structure of distributive lattice. Afterwards, we prove that the same method gives similar results for examples in spaces of tilings induced by tilings in the triangular lattice.

\section{Tiling groups and tiling functions}

\section{$2.1 \quad$ Tilings}

let $\Lambda$ be the square lattice of the Euclidean plane. A (finite) figure $F$ of $\Lambda$ is a (finite) union of closed square cells of $\Lambda$. A figure $F$ is simply connected if $F$ and its complement $R^{2}-F$ both are connected. A finite simply connected figure $F$ is called a polygon of $\Lambda$. The boundary of a polygon $P$ canonically induces a cycle in $\Lambda$, which is called the boundary cycle of $P$. A set $S$ of prototiles is a fixed finite set of polygons of $\Lambda$. A tile is a translated copy of a prototile. A tiling $T$ of a figure $F$ is a set of tiles included in $F$, with pairwise disjoint interiors, such that the union of the tiles of $T$ equals $F$.

\subsection{Groups and their representation}

let $\Sigma$ be the set $\left\{a, b, a^{-1}, b^{-1}\right\}, F_{a, b}$ be the free group generated by $a, b$ and $\pi$ denote the canonical surjection from the language $\Sigma^{*}$ of words with letters in $\Sigma$ to $F_{a, b}$. 
Let $R=\left\{r_{1}, r_{2}, \ldots, r_{p}\right\}$ be a finite set of words of $\Sigma^{*}$. The group $N_{R}$ denotes the normal group of $F_{a, b}$ generated by the elements of $\pi(R)$ and $\left\langle a, b \mid r_{1}, r_{2}, \ldots, r_{p}\right\rangle$ denotes the quotient group $F_{a, b} / N_{R}$. The group $<a, b \mid r_{1},, r_{2}, \ldots, r_{p}>$ has a classical graphic representation : the Cayley graph $C_{R}$ is the directed graph with labeled edges with labels in $\{a, b\}$ such that:

- vertices of $C_{R}$ are elements of $\left\langle a, b \mid r_{1}, r_{2}, \ldots, r_{p}\right\rangle$,

- the set of labels is $\{a, b\}$,

- for each 3-uple $\left(g, g^{\prime}, u\right)$ of elements of $\left(<a, b \mid r_{1}, r_{2}, \ldots, r_{p}>\right)^{2} \times\{a, b\}$, $g u=g^{\prime}$ if and only if there exists an edge of $C_{R}$ from $g$ to $g^{\prime}$, labeled by $u$.

Hence, the underlying graph of $\Lambda$ can be seen as the Cayley graph $C_{c e l l}=$ $C_{R_{0}}$, with $R_{0}=\left\{a b a^{-1} b^{-1}\right\}$, each element of $\{a, b\}$ being associated to a unit move ( $a$ for a horizontal rightwards move, $b$ for a vertical upwards move). By this way, each vertex of $\Lambda$ is identified to an element of $\left\langle a, b \mid a b a^{-1} b^{-1}\right\rangle$.

\subsection{Tiling groups}

Let $\mu=\left(v_{0}, v_{1}, \ldots, v_{p^{\prime}}\right)$ be a path of $\Lambda$, i. e. a sequence of vertices such that, for each integer $i$, with $0 \leq i<p^{\prime}$, there exists an element of $\Sigma$ such that $v_{i} u_{i}=v_{i+1}$. The path word $w(\mu)$ is the word $u_{0} u_{1} \ldots u_{p^{\prime}-1}$. Moreover, if $\mu$ is a boundary cycle of a polygon $P$, we say that $w(\mu)$ is a contour word of $P$.

Let $S=\left\{t_{1}, t_{2}, \ldots, t_{p}\right\}$ be a set of prototiles, and let $R=\left\{r_{1}, r_{2}, \ldots, r_{p}\right\}$ be a set of words such that for each integer $i$ such that $1 \leq i \leq p, w_{i}$ is a contour word of $t_{i}$. The tiling group of $S$ is the group $G_{t i l e}=<a, b \mid r_{1}, r_{2}, \ldots, r_{p}>$, and the tiling Cayley graph of $S$ is the graph $C_{\text {tile }}=C_{R}$.

Remark that tiling groups and tiling Cayley graphs only depend on the set $S$, and not on the contour words chosen for each prototile.

For $S_{0}=\left\{c_{0}\right\}$, where $c_{0}$ denotes the unit cell of $\Lambda$, the tiling group $G_{c e l l}$ of $S_{0}$ is isomorphic to $Z^{2}$ and can be identified to $\Lambda$.

\subsection{Tiling functions}

let $T$ be a tiling of a figure $F$. The graph $G_{T}$ of $T$ is the subgraph of $\Lambda$ (= $G_{\text {cell }}=Z^{2}$ ) generated by the set of edges which are on boundaries of tiles of $T$ (i. e. which cut no tile of $T$ ).

Definition 1 let $T$ be a tiling of a figure $F$. A tiling function induced by $T$ is a mapping $f_{T}$ from the set $V_{T}$ of vertices of $G_{T}$ to $G_{\text {tile }}$ such that, for each pair $(v, u)$ of $V_{T} \times\{a, b\}$, if the edge outgoing from $v$ labeled by $u$ is an edge of $G_{T}$, then the equality : $f_{T}(v u)=f_{T}(v) u$ holds.

Proposition 1 (J. H. Conway) let $F$ be a figure of $\Lambda, T$ be a tiling of $F$, $v_{0}$ be a vertex of $G_{T}$ and $v_{0}^{\prime}$ be a vertex of $G_{\text {tile }}$.

If $F$ is connected (respectively is a polygon), then there exists at most one (respectively exactly one) tiling function $f_{T}$ induced by $T$ such that $f_{T}\left(v_{0}\right)=v_{0}^{\prime}$. 
Proof. : (sketch) function $f_{T}$ can easily be constructed successively exploring the contour of each tile : function $f_{T}$ is first defined on the vertices of a tile $t_{0}$ which has $v_{0}$ on its boundary. Afterwards, $f_{T}$ is defined on the vertices of a tile $t_{1}$ which has a common vertex with $t_{0}$ and so on.

This method gives the uniqueness of $f_{T}$, for $F$ connected. Nevertheless, a conflict (i. e. a vertex $v$ such that two distinct values of $f_{T}(v)$ ) can arise if $F$ has some holes, which yields that there is no tiling function.

Remark 1 : let $P$ be a polygon and $v_{0}$ be a vertex of the boundary of $P$. If $f$ and $f^{\prime}$ are tiling functions such that $f\left(v_{0}\right)=f^{\prime}\left(v_{0}\right)$, then for each vertex $v$ of the boundary of $P, f(v)=f^{\prime}(v)$.

The use of tiling functions is a main method to study tilings. Interesting examples are developed in [1], [2], [3], [4], [5], [9].

We apply the theoretical notions on our special case below.

\section{Groups for tilings with bars}

Let $m$ and $n$ being fixed positive integers such that $m \geq 2$ and $n \geq 2$. The first sets of prototiles on which we apply notions of the previous section is (as in [2]) the set $S_{m, n}=\left\{h_{m}, v_{n}\right\}$, where

- the prototile $h_{m}$ denotes an $m \times 1$ horizontal rectangle, which admits $a^{m} b a^{-m} b^{-1}$ for contour word,

- the prototile $v_{n}$ denotes a $1 \times n$ vertical rectangle which admits $b^{n} a b^{-n} a^{-1}$ for contour word.

Thus, a set $R_{b a r s}$ of contour words of prototiles is $\left\{a^{m} b a^{-m} b^{-1}, b^{n} a b^{-n} a^{-1}\right\}$, which gives a group $G_{b a r s}=<a, b \mid a^{m} b a^{-m} b^{-1}, b^{n} a b^{-n} a^{-1}>$. Since this group has a complex structure, quotients groups of $G_{b a r s}$ will be used, in order to have groups that can be easily described. This is an indirect way to understand the structure of $G_{\text {bars }}$.

\subsection{Quotient groups}

To obtain such quotient groups, it suffices to exhibit a set $R^{\prime}=\left\{r_{1}^{\prime}, r_{2}^{\prime}, \ldots, r_{p^{\prime}}^{\prime}\right\}$ of words such that the words of $R_{\text {bars }}$ are null in $\left\langle a, b \mid r_{1}^{\prime}, r_{2}^{\prime}, \ldots, r_{p^{\prime}}^{\prime}\right\rangle$. In this case, we have a natural surjection from $G_{b a r s}$ to $\left\langle a, b \mid r_{1}^{\prime}, r_{2}^{\prime}, \ldots, r_{p^{\prime}}^{\prime}\right\rangle$.

\subsubsection{The cycle group}

As in [2], the principal quotient group used is constructed from the auxiliary set $R^{\prime}=\left\{a^{m}, b^{n}\right\}$. Obviously, the elements of $R_{b a r s}$ are null in $\left\langle a, b \mid a^{m}, b^{n}\right\rangle$, which guarantees that we have a canonical surjection $s$ from $G_{b a r s}$ to $<$ $a, b \mid a^{m}, b^{n}>$.

The structure of $\left\langle a, b \mid a^{m}, b^{n}\right\rangle$ is rather simple: it is isomorphic to the free product of a cyclic group of $m$ elements and a cyclic group of $n$ elements. The associated Cayley graph $C_{R^{\prime}}$ is formed from directed cycles of length $m$ with edges labeled by $a$ and directed cycles of length $n$ with edges labeled by $b$, each vertex being element of exactly two cycles, one of each type (see figure 1). 


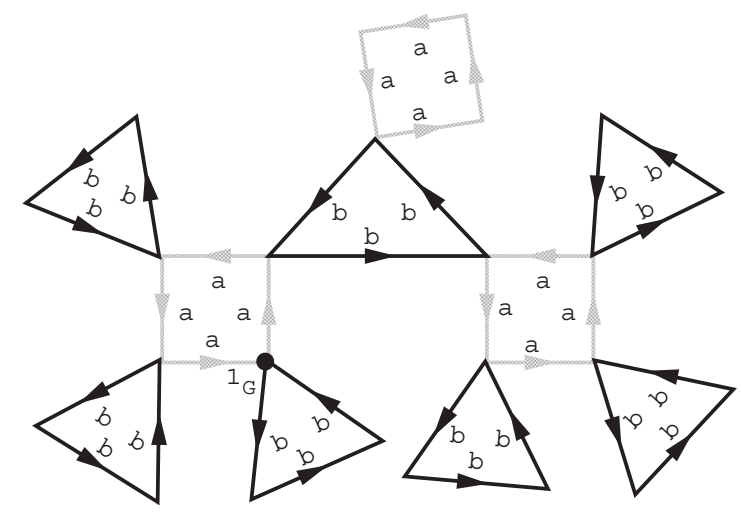

Figure 1: The Cayley graph of the group $G_{\text {cycles }}$ (example with $m=4$ and $n=3)$.

Moreover, $C_{R^{\prime}}$ is a tree of cycles : the only cycles of $C_{R^{\prime}}$ are those described above. Thus we state $C_{R^{\prime}}=C_{\text {cycles }}$ and $\left\langle a, b \mid a^{m}, b^{n}\right\rangle=G_{\text {cycles }}$.

We introduce some definitions which permit us to have a geometrical understanding of $G_{\text {cycles }}$.

Definition 2 (canonical expression, length, distance, order in the cycle group) For each element $w$ of $G_{\text {cycles }}$, there exists a unique finite sequence $\left(x_{1}, i_{1}, x_{2}, i_{2}, \ldots, x_{p}, i_{p}\right)$ such that :

- for each integer $j, x_{j}$ is element of $\{a, b\}$, and (for $j<p$ ), $x_{j} \neq x_{j+1}$,

- the equality : $w=x_{1}^{i_{1}} x_{2}^{i_{2}} \ldots x_{p}^{i_{p}}$, holds in $G_{\text {cycles }}$,

- if $x_{j}=a$ (respectively $x_{j}=b$ ), then $1 \leq i_{j}<m$ (respectively $1 \leq i_{j}<n$ ).

With these notations, we say that:

- the word $x_{1}^{i_{1}} x_{2}^{i_{2}} \ldots x_{p}^{i_{p}}$ is the canonical expression of $w$,

- the integer $p$ is the length of $w$ (denoted by $l(w)$ ),

- for $p \geq 2$, the element $x_{p-1}^{i_{p-1}} x_{p}^{i_{p}}$ of $G_{\text {cycles }}$ is called the final part of $w$ (denoted by $\operatorname{fin}(w)$ ) ; otherwise, for $p \leq 1$, we state $\operatorname{fin}(w)=w$,

- for $p \geq 2$, the element $x_{1}^{i_{1}} x_{2}^{i_{2}}$ of $G_{\text {cycles }}$ is called the initial part of $w$ (denoted by init $(w)$ ) ; otherwise, for $p \leq 1$, we state init $(w)=w$,

- the distance $d\left(w^{\prime}, w^{\prime \prime}\right)$ between two elements of $G_{c y c l e s}$ is equal to $l\left(w^{\prime-1} w^{\prime \prime}\right)$,

- for each integer $p^{\prime}$ such that $0 * \leq p^{\prime}$, the word $x_{1}^{i_{1}} x_{2}^{i_{2}} \ldots x_{p^{\prime}}^{i_{p^{\prime}}}$ is called a well formed prefix of the canonical expression of $w$,

- if $w^{\prime}$ and $w "$ are elements of $G_{\text {cycles }}$ such that the canonical expression of $w^{\prime}$ is a well formed prefix of the canonical expression of $w^{\prime}$, then $w^{\prime} \leq_{\text {cycles }}$ w". 
Remark 2 The relation $\leq_{\text {cycles }}$ defined above is obviously an order relation such that each element $w$ of $G$ (such that $w \neq 1_{G_{\text {cycles }}}$ ) has a unique immediate predecessor, denoted by $\operatorname{pr}_{\text {cycles }}(w)$. In other words, the relation $\leq_{c y c l e s}$ induces a structure of tree on $G_{\text {cycles }}$.

Thus, for each pair for each pair $\left(w^{\prime}, w\right.$ ") of elements of $G_{c y c l e s}$, there exists an element inf $\left(w^{\prime}, w^{\prime \prime}\right)$ in $G_{\text {cycles }}$ (i. e. in $f_{\text {cycles }}\left(w^{\prime}, w^{\prime \prime}\right)$ is such that in $f_{\text {cycles }}\left(w^{\prime}, w^{\prime \prime}\right) \leq_{\text {cycles }} w^{\prime}$, in $f_{\text {cycles }}\left(w^{\prime}, w^{\prime \prime}\right) \leq_{\text {cycles }} w^{\prime}$ and for each element $w^{\prime \prime \prime}$ such that $w^{\prime \prime \prime} \leq_{\text {cycles }} w^{\prime}$ and $w^{\prime \prime \prime} \leq_{\text {cycles }} w^{\prime \prime}$, we have : $w^{\prime \prime \prime} \leq_{\text {cycles }}$ inf $f_{\text {cycles }}\left(w^{\prime}, w^{\prime \prime}\right)$.

\subsubsection{The cell group}

Another quotient group of $G_{b a r s}$ is the group $G_{c e l l}$ defined from the set $R_{0}=$ $\left\{a b a^{-1} b^{-1}\right\}$. As we have seen before, this group isomorphic to $Z^{2}$.

\subsubsection{The torus group}

The third quotient group which will be used is $G_{\text {torus }}=<$ $a, b \mid a^{m}, b^{n}, a b a^{-1} b^{-1}>$, constructed using the set $R^{\prime \prime}=R^{\prime} \cup R_{0}=$ $\left\{a^{m}, b^{n}, a b a^{-1} b^{-1}\right\}$. This group is isomorphic to $Z_{m} \times Z_{n}$, i. e. the direct product of a cyclic group of $m$ elements and a cyclic group of $n$ elements : each element can be seen as a pair $(i, j)$ of $Z_{m} \times Z_{n}$. The associated Cayley graph $C_{R}$ " is formed from directed cycles of length $m$ with edges labeled by $a$ and directed cycles of length $n$ with edges labeled by $b$, in such a way that $a$ and $b$ commute. It has the structure of a torus $T_{m \times n}$. Notice that $G_{t o r u s}$ is also a quotient group of both $G_{\text {cycles }}$ and $G_{c e l l}$.

\subsection{Bar tiling projections}

Let $T$ be a tiling of a polygon $P$, and $f_{T}$ be a tiling function induced by $T$. Function $g_{T}$, defined by : $g_{T}=\pi^{\prime} o f_{T}$, (where $\pi^{\prime}$ denotes the canonical surjection from $G_{\text {bars }}$ to $G_{\text {cycles }}$ and $o$ denotes the composition of functions) is called a tiling projection of $T$. Notice that, for a set of bars as set of prototiles, the set of vertices of $G_{T}$ is the set of vertices which are elements of $P$ (since each vertex of $P$ is on the boundary of a bar of $T$ ).

Fix a vertex $v_{0}$ of the boundary of $P$ and assume that $g_{T}\left(v_{0}\right)=1_{G_{c y c l e s}}$. Let $v$ be any vertex of $P$; how can we compute $g_{T}(v)$ ? we have to find a path of $P$ from $v_{0}$ to $v$ which cuts no tile of $T$, and, from the definitions of $f_{T}$ and $g_{T}$, the word associated to this path, seen as an element of $G_{c y c l e s}$, is equal to $g_{T}(v)$ (see Figure 2).

Remark 3 Let $\left(T, T^{\prime}\right)$ be a pair of tilings of $P$, and $v$ be a vertex of the boundary of $P$. taking a path from $v_{0}$ to $v$ on the boundary of $P$, we obtain : $g_{T}(v)=$ $g_{T^{\prime}}(v)$. In other words, the value $g_{T}(v)$ does not depend on the tiling chosen.

Remark 4 Let $\left(v, v^{\prime}\right)$ be a pair of neighbor vertices of $P$. If the edge which join them is on the boundary of a bar, then $d\left(g_{T}(v), g_{T}\left(v^{\prime}\right)\right)=1$. If, otherwise, this edge cuts a bar, then $d\left(g_{T}(v), g_{T}\left(v^{\prime}\right)\right)=3$. Thus function $g_{T}$ completely gives the tiling $T$. 

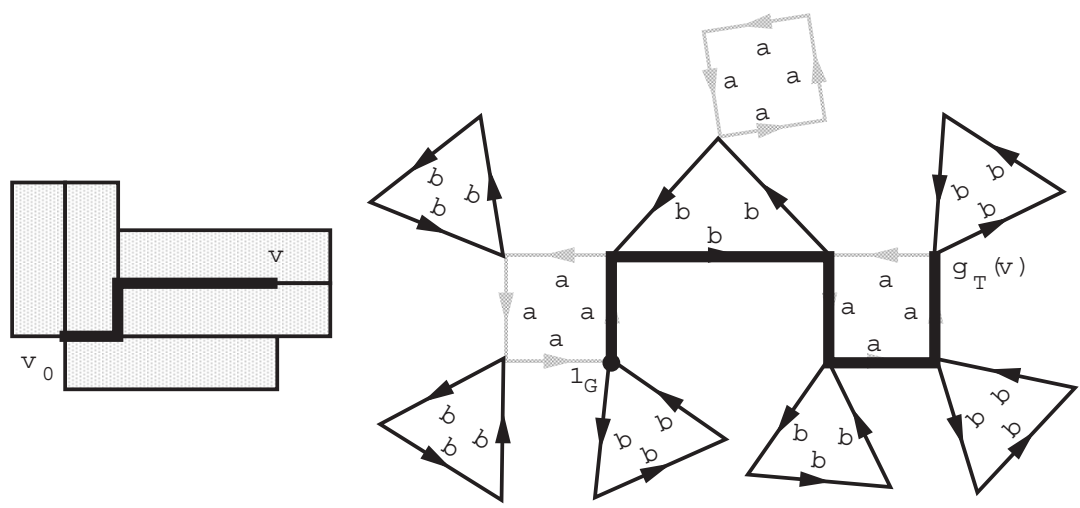

Figure 2: Computation of $g_{T}(v)$

Definition 3 For each element $v$ of the planar grid, there exists a unique pair $\left(i_{v}, j_{v}\right)$ of $Z^{2}$ such that $v=v_{0} a^{i_{v}} b_{j_{v}}$. We define cong $g_{\text {torus }}(v)$ as the element of $G_{\text {torus }}$ such that:

$$
\operatorname{cong}_{\text {torus }}(v)=a^{i_{v}} b_{j_{v}}
$$

Hence, cong $g_{\text {torus }}$ is a function from the planar grid to $G_{\text {torus }}$.

Remark 5 Let $s$ denote the canonical surjective morphism from $G_{c y c l e s}$ to $G_{\text {torus. }}$. One obviously sees (by induction on the length of a shortest path, from $v_{0}$ to $v$, which cut no tile) that, for each tiling $T$ and each vertex $v$ of $P$, we have : $s\left(g_{T}(v)\right)=\operatorname{cong}_{\text {torus }}(v)$.

Thus, for each pair $\left(T, T^{\prime}\right)$ of tilings of $P$ and each vertex $v$ of $P$, we have

$$
g_{T}(v)^{-1} g_{T^{\prime}}(v) \in \operatorname{ker}(s)
$$

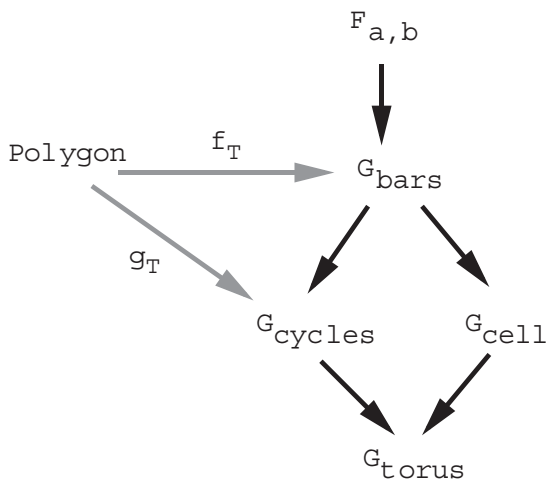

Figure 3: mappings used (dark arrows represent canonical surjective group morphisms)

The proposition below gives a characterization of tiling projections, from a congruence condition and a local Lipschitz condition. It gives an algebraic interpretation of the the geometrical regularity of a tiling. 
Proposition 2 Let $g$ be a function from the set of vertices of $P$ to $G_{c y c l e s}$ such that :

- $g\left(v_{0}\right)=1_{G_{c y c l e s}}$,

- for each vertex vertex $v$ of $P, s(g(v))=\operatorname{cong}_{\text {torus }}(v)$,

- for each pair $\left(v, v^{\prime}\right)$ of neighbors vertices of $P, d\left(g(v), g\left(v^{\prime}\right)\right) \leq 3$, and if, moreover, the line segment $\left[v, v^{\prime}\right]$ is included on the boundary of $P$, then $d\left(g(v), g\left(v^{\prime}\right)\right)=1$.

There exists a tiling $T$ of $P$ such that $g=g_{T}$.

Proof. Let $v$ and $v^{\prime}$ be vertices of the polygon $P$ such that $v^{\prime}=v a$ (respectively $\left.v^{\prime}=v b\right)$. Let $u$ be the element of $G_{\text {cycles }}$ such that $g\left(v^{\prime}\right)=g(v) u$. We necessarily have $s(u)=a$ (respectively $s(u)=b$ ). Thus, since $l(u) \leq 3$, there exists an integer $j$, with $0 \leq j<n$, such that $u=b^{j} a b^{-j}$ (respectively there exists an integer $i$, with $0 \leq i<m$, such that $\left.u=a^{i} b a^{-i}\right)$.

We claim the following fact (we can also claim the symmetric fact) :

Fact : Assume that $v^{\prime}=v a$ and there exists an integer $j$, with $0<j<n$ such that $g\left(v^{\prime}\right)=g(v) b^{j} a b^{-j}$. Then, for each integer $j^{\prime}$ such that $j-n \leq j^{\prime} \leq j$, we have : $g\left(v b^{j^{\prime}}\right)=g(v) b^{j^{\prime}}$ and $g\left(v^{\prime} b^{j^{\prime}}\right)=g\left(v^{\prime}\right) b^{j}$

We prove this fact as follows : first notice that the cells an edge of which is the line segment $\left[v, v^{\prime}\right]$ are included in $P$, since $d\left(g(v), g\left(v^{\prime}\right)\right) \neq 1$. Thus, the vertices $v b$ and $v^{\prime} b$ both are in $P$.

Now let $u^{\prime}$ and $u$ " such that $g(v b)=g(v) u^{\prime}$ and $g\left(v^{\prime} b\right)=g\left(v^{\prime}\right) u "$. There exists a pair $\left(i, i^{\prime}\right)$ of $\{0,1, \ldots, m-1\}^{2}$ such that $u^{\prime}=a^{i^{\prime}} b a^{-i^{\prime}}$ and $u^{\prime \prime}=$ $a^{i \prime} b a^{-i^{\prime \prime}}$. With these notations, using the path $\left(v b, v, v^{\prime}, v^{\prime} b\right)$, we have $g\left(v^{\prime} b\right)=$ $g(v b)\left(u^{\prime}\right)^{-1} b^{j} a b^{-j} u$ ". From our hypothesis, we have $l\left(\left(u^{\prime}\right)^{-1} b^{j} a b^{-j} u^{\prime \prime}\right) \leq 3$, which necessarily yields $i^{\prime}=i "=0$, since $j \neq 0$.

We have obtained the claim for $j^{\prime}=1$, and, moreover, $g\left(v^{\prime} b\right)=$ $g(v b) b^{j-1} a b^{-j+1}$. Thus, if $j-1 \neq 0$, we can repeat the same argument for $g\left(v^{\prime} b^{2}\right)$ and $g\left(v b^{2}\right)$, and so on for $g\left(v b^{j^{\prime}}\right)$ and $g\left(v b^{j^{\prime}}\right)$, while $j^{\prime} \leq j$. We can also use the same argument in the other direction (for $j^{\prime}<0$ ) while $j^{\prime} \geq j-n$, which concludes the proof of the fact.

We now introduce the set $T$ of tiles defined as follows : a vertical (respectively horizontal) bar is in $T$ if and only if there exists a vertex $v$ of $P$ and an integer $j$ with $0<j<n$ (respectively an integer $i$ with $0<i<m$ ), such that $g(v a)=g(v) b^{j} a b^{-j}$ (respectively $\left.g(v b)=g(v) a^{i} b a^{-i}\right)$.

There is no overlap, from the fact above. Let $\left(v_{0}, v_{1}, v_{2}, v_{3}, v_{4}=v_{0}\right)$ be a contour cycle of a cell, in the trigonometric sense, such that $v_{0}$ is the south west corner of the cell. If we have $d\left(g\left(v_{0}\right), g\left(v_{1}\right)\right)=d\left(g\left(v_{1}\right), g\left(v_{2}\right)\right)=d\left(g\left(v_{2}\right), g\left(v_{3}\right)\right)=$ $d\left(g\left(v_{3}\right), g\left(v_{4}\right)\right)=1$, then we have $g\left(v_{0}\right)=g\left(v_{0}\right) a b a^{-1} b^{-1}$, which is a contradiction. Thus there exists an integer $i$ of $\{0,1,2,3\}$ such that $d\left(g\left(v_{i}\right), g\left(v_{i+1}\right)\right)=3$ , which guarantees that the cell is covered by a bar of $T$. Thus there is no gap and $T$ is actually a tiling of $P$.

The fact that $g_{T}=g$ is obvious, from the definition of $T$ and the fact above, which concludes the proof. 


\subsection{The kernel of the morphism from $G_{\text {cycles }}$ to $G_{\text {torus }}$}

Let $s$ denote the canonical surjective morphism from $G_{\text {cycles }}$ to $G_{\text {torus }}$. We will see that the subgroup $\operatorname{ker}(s)$ of $G_{\text {cycles }}$ has a fundamental importance in the comparison of tilings. This is a consequence of remark 5 .

We have to explore the structure of this group.

Let $S_{\text {rect }}$ be the subset of $G_{\text {cycles }}$ defined by $S_{\text {rect }}=\left\{a^{i} b^{j} a^{-i} b^{-j}, b^{j} a^{i} b^{-i} a^{-j}\right.$, for $1 \leq i<m$ and $1 \leq j<n\}$. One easily verifies that $S_{r e c t}$ is closed by inverse (i. e. if $t \in S_{\text {rect }}$, then $\left.t^{-1} \in S_{\text {rect }}\right)$ and $S_{\text {rect }} \subset \operatorname{ker}(s)$.

Remark 6 Let $w$ be an element of kers $(s)$. By projection (since $G_{\text {torus }}$ is commutative), one obviously verifies that $l(w) \leq 4$ if and only if $w \in S_{\text {rect }} \cup$ $\left\{1_{G_{\text {cycles }}}\right\}$.

We need the following result to study the space of tilings of $P$ with bars :

Proposition 3 Let $w$ be an element of $k e r(s)$. There exists a unique finite sequence $\left(t_{1}, t_{2}, \ldots, t_{p}\right)$ of elements of $S_{\text {rect }}$ (called the decomposition of $\left.w\right)$, such that $w=\Pi_{i=1}^{p} t_{i}$ and, for each integer $i$ such that $0<i<p, t_{i} t_{i+1} \neq 1_{G_{c y c l e s}}$.

Moreover, the decomposition of $w$ can be computed in $O(l(w))$ time units.

We decompose the proof of the above proposition into two lemmas :

Lemma 1 (existence and computation of a decomposition) Let $w$ be an element of $\operatorname{ker}(s)$ (different from $1_{G_{\text {cycles }}}$ ) such that the initial part of $w$ is $a^{i} b^{j}$ (respectively $b^{j} a^{i}$ ). We state : $w=a^{i} b^{j} a^{-i} b^{-j} w^{\prime}$ (respectively $w=$ $\left.b^{j} a^{i} b^{-j} a^{-i} w^{\prime}\right)$.

We have $: l\left(w^{\prime}\right) \leq l(w)-1$

Proof. Assume that the the initial part of $w$ is $a^{i} b^{j}$. From remark 6, we can state $w=a^{i} b^{j} a^{k} u$, with $l(u)=l(w)-3$ and the canonical expression of $u$ begins by $b$.

Thus $w^{\prime}=b^{j} a^{k-i} u$, which gives $l\left(w^{\prime}\right) \leq l(u)+2=l(w)-1$.

The symmetric case can be treated in a symmetric way.

Lemma 2 (unicity of the decomposition) Let $\left(t_{1}, t_{2}, \ldots, t_{p}\right)$ be a (nonempty) sequence of elements of $S_{\text {rect }}$ such that, for each integer $i$ such that $0<i<p, t_{i} t_{i+1} \neq 1$. Let $w$ be defined by $w=\Pi_{i=1}^{p} t_{i}$. Then :

- $l(w) \geq p+3$,

- $t_{p}=a^{-i} b^{-j} a^{i} b^{j}$ (respectively $t_{p}=b^{-j} a^{-i} b^{j} a^{i}$ ) if and only if the final part of $w$ is $a^{i} b^{j}$ (respectively $b^{j} a^{i}$ ).

Proof. By induction on the integer $p$. The result is obvious if $p=1$. Assume that the result is true for each element $w$ such that $w$ is a product of $p$ elements of $S_{\text {rect }}$. Let $w^{\prime}$ be an product of $p+1$ elements of $S_{\text {rect }}$. we state $w^{\prime}=t_{1} t_{2} \ldots t_{p} t_{p+1}$. By induction hypothesis, if $t_{p}=a^{-i} b^{-j} a^{i} b^{j}$, the canonical expression of element $w=t_{1} t_{2} \ldots t_{p}$ is of type $u a^{i} b^{j}$, with $u$ such that $l(u) \geq p+1$ and the canonical expression of $u$ finishes by $b$. 
If $t_{p+1}=a^{-k} b^{-l} a^{k} b^{l}$, then the canonical expression of $w^{\prime}$ is $u a^{i} b^{j^{\prime}} a^{m-k} b^{n-l} a^{k} b^{l}$, which gives the first item and the direct part of the second item of the lemma.

If $t_{p+1}=b^{-l} a^{-k} b^{l} a^{k}$, then we have $w^{\prime}=u a^{i} b^{j-l} a^{-k} b^{l} a^{k}$. If $j \neq l$, then the results of the lemma hold. If $j-l=0$, then we have $w^{\prime}=u a^{i-k} b^{l} a^{k}$. Notice that if $i=k$, then $t_{p} t_{p+1}=1$ which is a contradiction. Thus $a^{i-k} \neq 1$, which gives the first item and the direct part of the second item of the lemma.

Conversely, if the final part of $w^{\prime}$ is $a^{l} b^{k}$, then we necessarily have $t_{p+1}=$ $b^{-l} a^{-k} b^{l} a^{k}$, since, otherwise, the direct part of the second item of the lemma would be contradicted.

The symmetric cases can be treated in a similar way.

The proposition proved above allows the definitions below :

Definition 4 (decomposition number of an element of $k e r(s)$ ) The decomposition number of an element $w$ of $\operatorname{ker}(s)$ (denoted by num $(w)$ ) is the number of factors of its decomposition.

Definition 5 (order on $\operatorname{ker}(s)$ ) Let $w$ and $w^{\prime}$ be elements of ker $(s)$ whose decompositions are $w=\Pi_{i=1}^{p} t_{i}$ and $w^{\prime}=\Pi_{i=1}^{p^{\prime}} t_{i}^{\prime}$. We say that $w \leq_{\text {decomp }} w^{\prime}$ if $p \leq p^{\prime}$ and for each integer $i$ such that $1 \leq i \leq p$, we have $t_{i}=t_{i}^{\prime}$

Remark 7 The relation defined by this way is obviously an order relation on the set $\operatorname{ker}(s)$. Moreover, each element $w$ of $\operatorname{ker}(s)$ (such that $w \neq 1_{G_{\text {cycles }}}$ ) has a unique immediate predecessor (denoted by $\operatorname{pr}_{\text {decomp }}(w)$ ) for this relation. In other words, the relation defined induces a structure of directed tree on $\operatorname{ker}(s)$.

Thus, for each pair $\left(w, w^{\prime}\right)$ of elements of ker $(s)$, there exists an element $w "$ such that $w "=i n f_{\text {decomp }}\left(w, w^{\prime}\right)$. Notice that in $f_{\text {decomp }}\left(w, w^{\prime}\right)$ is the unique element w" of $\operatorname{ker}(s)$ such that :

$i n f_{\text {decomp }}\left(w^{\prime-1} w, w^{\prime-1} w^{\prime}\right)=i n f_{\text {decomp }}\left(w^{\prime-1}, w^{\prime-1} w\right)=i n f_{\text {decomp }}\left(w^{\prime-1}, w^{\prime-1} w^{\prime}\right)=1_{G_{\text {cycles }}}$

From Lemma 1, we have the following remark :

Remark 8 For each element $w$ of $\operatorname{ker}(s)$ (such that $\left.w \neq 1_{G_{\text {cycles }}}\right), p r_{\text {decomp }}(w)$ is the unique element $w^{\prime}$ such that there exists an element $t_{i}$ of $S_{\text {rect }}$ such that $w^{\prime}=w t_{i}$ and $l\left(w^{\prime}\right) \leq l(w)-1$

Moreover, for each element $t_{j}$ of $S_{\text {rect }}$ such that $t_{j} \neq t_{i}$, we have $l\left(w t_{j}\right) \geq$ $l(w)+1$

\section{Distance between tilings}

Definition 6 (distance between two tilings) Let $\left(T, T^{\prime}\right)$ be a pair of tilings of $P$, and let $g_{T}$ and $g_{T^{\prime}}$ be the associated projections (such that $g_{T}\left(v_{0}\right)=$ $\left.g_{T^{\prime}}\left(v_{0}\right)=1_{G_{\text {cycles }}}\right)$. The distance $\Delta\left(T, T^{\prime}\right)$ is defined by the equality :

$$
\Delta\left(T, T^{\prime}\right)=\sum_{v \in P} n u m\left(g_{T^{\prime}}(v)^{-1} g_{T}(v)\right)
$$

We will prove that the distance defined above has a geometric interpretation, using the local flips defined below. 


\subsection{Local flips}

Let $T$ be a tiling of $P$. Assume that there exists an $m \times n$ rectangle $R_{0}$ such that $T$ contains a tiling $T_{0}$ of $R_{0}$ (in this case, tiles of $T_{0}$ are copies of the same prototile). Another tiling $T_{\text {flip }}$ of $P$ is obtained replacing tiles of $T_{0}$ by tiles of $T_{1}$, where $T_{1}$ denotes the only tiling of $R_{0}$ different from $T_{0}$. We say that $T_{\text {flip }}$ is deduced from $T$ by a local flip whose support is $R_{0}$ (see figure 4 ).
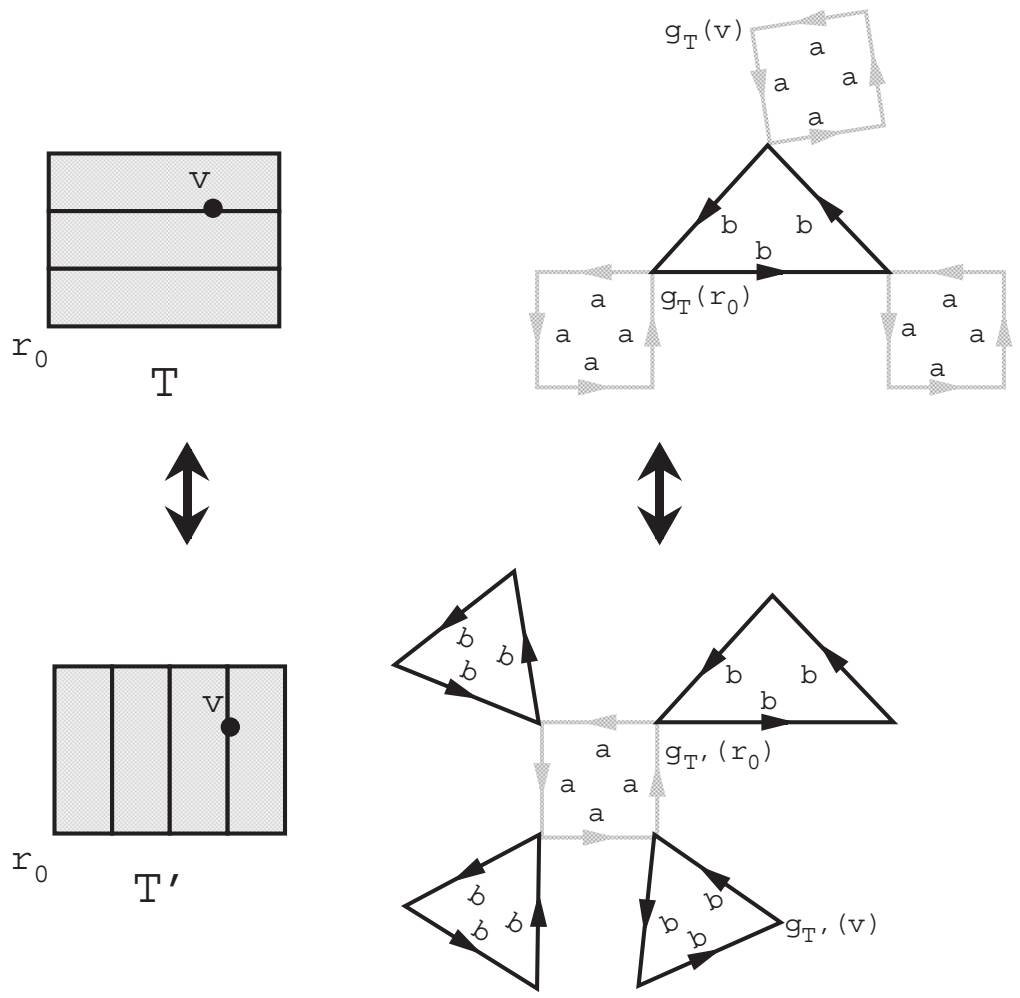

Figure 4: Local flips for bars.

If $v$ is a vertex of $P$ which is not in the interior of $R_{0}$, then there exists a path from $v_{0}$ to $v$ which cuts not tile of $T$ and does not go through the interior part of $R_{0}$. This path also cuts not tile of $T_{f l i p}$. Thus, $g_{T}(v)=g_{T_{f l i p}}(v)$.

If $v$ is an interior vertex of $R_{0}$, let $r_{0}$ be the lower left corner of $R_{0}$. Let state $v=r_{0} a^{i} b^{j}\left(=r_{0} b^{j} a^{i}\right)$. Assume that $T_{0}$ is formed from $h_{m}$ tiles. Then we have $g_{T}(v)=g_{T}\left(r_{0}\right) b^{j} a^{i}$ and $g_{T_{f l i p}}(v)=g_{T}\left(r_{0}\right) a^{i} b^{j}$. Thus

$$
g_{T_{f l i p}}(v)=g_{T}(v) a^{-i} b^{-j} a^{i} b^{j}
$$

This equality means that local flip induces a multiplication of the tiling projection of each interior vertex of $R_{0}$ by an appropriate element of $S_{\text {rect }}$. Thus

$$
\Delta\left(T, T_{\text {flip }}\right)=(m-1)(n-1)
$$




\subsection{The flip formula}

It has been proved, as a consequence of the algorithm of tiling, [2] that for each pair $\left(T, T^{\prime}\right)$ of tilings of $P$, there exists a sequence of local flips which permit to deduce $T^{\prime}$ from $T$. We will improve this result, giving a formula for the minimal number $\min \operatorname{lip}\left(T, T^{\prime}\right)$ of necessary flips.

Proposition 4 For each pair $\left(T, T^{\prime}\right)$ of tilings of $P$, we have :

$$
\Delta\left(T, T^{\prime}\right) \leq(m-1)(n-1) \operatorname{minflip}\left(T, T^{\prime}\right)
$$

Proof. Let $\left(T=T_{0}, T_{1}, \ldots, T_{p}=T^{\prime}\right)$ be a sequence of tilings such that, for $0 \leq i<p, T_{i+1}$ is deduced from $T_{i}$ by a local flip. We have $\Delta\left(T, T^{\prime}\right) \leq$ $\sum_{i=0}^{p-1} \Delta\left(T_{i}, T_{i+1}\right)$.

From the study of local flips, we see that, for $0 \leq i<p, \Delta\left(T_{i}, T_{i+1}\right)=$ $(m-1)(n-1):$ if $v$ is not in the interior part of the rectangle on which the flip is done, then $g_{T_{i}}(v)=g_{T_{i+1}}(v)$, thus $\operatorname{num}\left(g_{T_{i}}(v)^{-1} g_{T_{i+1}}(v)\right)=0$; if $v$ is in the interior part of the rectangle on which the flip is done (we have $(m-1)(n-1)$ such interior vertices), then $g_{T_{i+1}}(v)$ is obtained multiplying $g_{T_{i}}(v)$ by an element of $S_{\text {rect }}$, thus $\operatorname{num}\left(g_{T_{i}}(v)^{-1} g_{T_{i+1}}(v)\right)=1$.

Thus $\Delta\left(T_{i}, T_{i+1}\right)=(m-1)(n-1)$, which yields that $\Delta\left(T, T^{\prime}\right) \leq(m-1)(n-$ 1) $p$, which gives the result.

We will prove that the inequality of the previous proposition is actually an equality. To do it, we have to exhibit a local flip in $T$ which decreases $\Delta\left(T, T^{\prime}\right)$ of $(m-1)(n-1)$ units. This flip will be done in the neighborhood of a special point which will be called a maximal vertex.

\subsubsection{Maximal vertex}

Definition 7 A maximal vertex for a pair $\left(T, T^{\prime}\right)$ of distinct tilings is a vertex $v$ of $P$ such that :

- $g_{T}(v) \neq g_{T^{\prime}}(v)$,

- $\sup \left[l\left(g_{T}(v)\right), l\left(g_{T^{\prime}}(v)\right)\right]$ is maximal with the previous condition.

There exists such a maximal vertex (since otherwise $g_{T}=g_{T^{\prime}}$, which yields that $T=T^{\prime}$ ). Let $v_{1}$ be a maximal vertex. It can be assumed without loss of generality that $l\left(g_{T^{\prime}}\left(v_{1}\right)\right) \leq l\left(g_{T}\left(v_{1}\right)\right)$.

Lemma 3 We have $l\left(g_{T}\left(v_{1}\right)\right) \geq 2$ and, moreover,

$$
\operatorname{fin}\left(g_{T}\left(v_{1}\right)\right)=\operatorname{fin}\left(g_{T^{\prime}}\left(v_{1}\right)^{-1} g_{T}\left(v_{1}\right)\right)
$$

Proof. We state $g_{T}\left(v_{1}\right)=u w$ and $g_{T^{\prime}}\left(v_{1}\right)=u w^{\prime}$, where $u$ denotes inf $f_{\text {cycles }}\left(g_{T}\left(v_{1}\right), g_{T^{\prime}}\left(v_{1}\right)\right)$. Thus, $g_{T^{\prime}}\left(v_{1}\right)^{-1} g_{T}\left(v_{1}\right)=w^{\prime-1} w$. Since it is assumed that $l\left(g_{T^{\prime}}\left(v_{1}\right)\right) \leq l\left(g_{T}\left(v_{1}\right)\right)$, we have $l\left(w^{\prime}\right) \leq l(w)$. Moreover, $4 \leq l\left(w^{\prime-1} w\right) \leq$ $l\left(w^{\prime}\right)+l(w)$, which yields that $2 \leq l(w)$

If $w=a^{i} b^{j}$, then we necessarily have $w^{\prime}=b^{j} a^{i}$ since $l\left(w^{\prime}\right) \leq l(w), w^{\prime} \neq w$ and $w^{\prime-1} w=g_{T^{\prime}}\left(v_{1}\right)^{-1} g_{T}\left(v_{1}\right)$ is element of $\operatorname{ker}(s)$. This fact gives the result (the same argument can be used in the symmetric case, when $w=b^{j} a^{i}$ ).

If $l(w) \geq 3$, then the result is obvious, since $i n f_{\text {cycles }}\left(w, w^{\prime}\right)=1_{G_{c y c l e s}}$. 
Proposition 5 Assume that the final part of $g_{T}\left(v_{1}\right)$ is $a^{i} b^{j}$ (respectively $b^{j} a^{i}$ ). Let $r_{0}$ be the vertex of the plane defined by $v_{1}=r_{0} a^{i} b^{j}$ (respectively $v_{1}=r_{0} b^{j} a^{i}$ ). This vertex is the lower left corner of an $m \times n$-rectangle $R_{0}$ such that $T$ contains a tiling $T_{R_{0}}$ of $R_{0}$ formed from vertical (respectively horizontal) tiles.

Proof. We treat the case when the final part of $g_{T}\left(v_{1}\right)$ is $a^{i} b^{j}$. Let $u_{1}$ be the element of $G_{\text {cycles }}$ such that $u_{1} a^{i} b^{j}=g_{T}\left(v_{1}\right)$. By definition of $g_{T}$, there exists a unique integer $l$, such that $0 \leq l<n$ and $g_{T}\left(v_{2}\right)=g_{T}\left(v_{1}\right) b^{-l} a b^{l}=u_{1} a^{i} b^{j} b^{-l} a b^{l}$. If we have $l \neq j$, then $l\left(g_{T}\left(v_{2}\right)\right)=l\left(g_{T}\left(v_{1}\right)\right)+2$, thus $l\left(g_{T}\left(v_{2}\right)\right)>l\left(g_{T}\left(v_{1}\right)\right)$.

Moreover, from the previous lemma, the canonical expression of $g_{T^{\prime}}\left(v_{1}\right)^{-1} g_{T}\left(v_{1}\right)$ is $w_{1} a^{i} b^{j}$, with $w_{1}$ finishing by $b$, thus $g_{T^{\prime}}\left(v_{1}\right)^{-1} g_{T}\left(v_{2}\right)=$ $w_{1} a^{i} b^{j} b^{-l} a b^{l}$. Thus, if $l \neq j$, then we have $d\left(g_{T}\left(v_{2}\right), g_{T^{\prime}}\left(v_{1}\right)\right)=$ $d\left(g_{T}\left(v_{1}\right), g_{T^{\prime}}\left(v_{1}\right)\right)+2$, which gives $d\left(g_{T}\left(v_{2}\right), g_{T^{\prime}}\left(v_{1}\right)\right) \geq 6$.

On the other hand, we have $d\left(g_{T^{\prime}}\left(v_{2}\right), g_{T^{\prime}}\left(v_{1}\right)\right) \leq 3$, which yields that $g_{T}\left(v_{2}\right) \neq g_{T^{\prime}}\left(v_{2}\right)$. The previous facts contradict the maximality of $v_{1}$, Thus we necessarily have $j=l$ and, consequently, $g_{T}\left(v_{2}\right)=g_{T}\left(v_{1}\right) b^{-j} a b^{j}$. This last equality implies (using the same kind of argument used in the proof of the fact of Proposition 2) that the vertical tile whose lower left corner is $r_{0} a^{i}$ is element of $T$.

If, moreover, $v_{2}$ is an interior vertex of $R_{0}$, then $i+1 \neq m$, thus $l\left(g_{T}\left(v_{2}\right)\right)=l\left(g_{T}\left(v_{1}\right)\right)$ and $d\left(g_{T}\left(v_{2}\right), g_{T^{\prime}}\left(v_{1}\right)\right)=d\left(g_{T}\left(v_{1}\right), g_{T^{\prime}}\left(v_{1}\right)\right) \geq 4$, which gives : $g_{T}\left(v_{2}\right) \neq g_{T^{\prime}}\left(v_{2}\right)$. Hence, $v_{2}$ is also a maximal vertex and we can repeat the argument for $v_{3}$, the right neighbor of $v_{2}$ and so on. The same kind of argument can also be used leftwards. This gives the tiling $T_{R_{0}}$ of $R_{0}$.

Proposition 6 Let $T_{\text {flip }}$ be the tiling deduced from $T$ by a flip on $R_{0}$. We have the equality: $\Delta\left(T_{\text {flip }}, T^{\prime}\right)=\Delta\left(T, T^{\prime}\right)-(m-1)(n-1)$.

Proof. Let state, for any vertex $v$ of the interior of $R_{0}, v=r_{0} a^{i^{\prime}} b^{j^{\prime}}\left(=r_{0} b^{j^{\prime}} a^{i^{\prime}}\right.$, and $g_{T^{\prime}}(v)^{-1} g_{T}(v)=\Pi_{i=1}^{p} t_{i}$. The final part of $g_{T}(v)$ is $a^{i^{\prime}} b^{j^{\prime}}$, which yields that the last factor of $g_{T^{\prime}}(v)^{-1} g_{T}(v)$ is $t_{p}=a^{-i^{\prime}} b^{-j^{\prime}} a^{i^{\prime}} b^{j^{\prime}}$. On the other hand,

$g_{T}(v)^{-1} g_{T_{f l i p}}(v)=\left(g_{T}(v)^{-1} g_{T}\left(v_{0}\right)\right)\left(g_{T}\left(v_{0}\right)^{-1} g_{T_{f l i p}}(v)\right)=b^{-j^{\prime}} a^{-i^{\prime}} b^{j^{\prime}} a^{i^{\prime}}=\left(t_{p}\right)^{-1}$

Thus,

$g_{T^{\prime}}(v)^{-1} g_{T_{f l i p}}(v)=\left(g_{T^{\prime}}(v)^{-1} g_{T}(v)\right)\left(g_{T}(v)^{-1} g_{T_{f l i p}}(v)\right)=\left(\Pi_{i=1}^{p} t_{i}\right)\left(t_{p}\right)^{-1}=\Pi_{i=1}^{p-1} t_{i}$

which yields that $: \operatorname{num}\left(g_{T^{\prime}}(v)^{-1} g_{T_{f l i p}}(v)\right)=\operatorname{num}\left(g_{T^{\prime}}(v)^{-1} g_{T}(v)\right)-1$, which gives the result.

Corollary 1 (flip formula) For each pair $\left(T, T^{\prime}\right)$ of tilings of $P$, we have:

$$
\Delta\left(T, T^{\prime}\right)=(m-1)(n-1) \operatorname{minflip}\left(T, T^{\prime}\right)
$$

Proof. obvious, by induction on $\Delta\left(T, T^{\prime}\right)$.

\subsection{Algorithm}

\subsubsection{Presentation}

The notion of maximal vertex permits to give an algorithm which, given a pair $\left(T, T^{\prime}\right)$ of tilings of a polygon gives a sequence $\left(R_{1}, R_{2}, \ldots, R_{\operatorname{minflip}\left(T, T^{\prime}\right)}\right)$ 
of rectangles on which flips can successively be done, to go from $T$ to $T^{\prime}$. Informally, such a sequence is a space economic way to encode a shortest path of tilings from $T$ to $T^{\prime}$. The algorithm is presented below :

Input : a pair $\left(T, T^{\prime}\right)$ of tilings of a same polygon $P$.

Initialization : Construct a spanning tree rooted in a fixed vertex $v_{0}$. When a new vertex $v$ is reached, compute the canonical expressions of $g_{T}(v)$, $g_{T^{\prime}}(v), g_{T^{\prime}}(v)^{-1} g_{T}(v)$, compute $\left.\sup \left(l\left(g_{T}\right)(v)\right), l\left(g_{T^{\prime}}(v)\right)\right)$, and place $v$ in a "list of lists" such that each vertex $v^{\prime}$ is in a basic list corresponding to the value $: \sup \left(l\left(g_{T}\left(v^{\prime}\right)\right), l\left(g_{T^{\prime}}\left(v^{\prime}\right)\right)\right)$, and those basic lists are ordered according to their decreasing corresponding values.

A variable list $L$ of rectangles stores the sequence of rectangles used. The beginning of this list is formed from rectangles which are supports of flips deduced from $T$ and the end of the list contains the rectangles which are supports of flips deduced from $T^{\prime}$. For initialization, $L$ is empty and each insertion is done just between both parts.

We also need a variable vertex $v_{1}$, which, for initialization, is the first element of the "list of lists" of vertices.

Repeat : Take the first element of the "list of lists" for $v_{1}$.

If $g_{T}\left(v_{1}\right)=g_{T^{\prime}}\left(v_{1}\right)$, then delete the value of $v_{1}$ from the "list of lists".

Otherwise, $v_{1}$ is a maximal vertex, which (in the case when $l\left(g_{T}\left(v_{1}\right)\right) \geq$ $l\left(g_{T^{\prime}}\left(v_{1}\right)\right)$, the symmetric case being treated in a symmetric way) is in a rectangle $R$ on which a flip of tiling $T$ can be done ( $R$ is defined by the final part of $g_{T}\left(v_{1}\right)$ ).

Insert this rectangle in $L$, and update replacing $T$ by $T_{f l i p}$ : for each vertex $v$ of the interior of $R$, update $g_{T}(v), \sup \left(l\left(g_{T}(v)\right), l\left(g_{T^{\prime}}(v)\right)\right)$ and the place of $v$ in the "list of lists" of vertices.

Notice that $l\left(g_{T}(v)\right)-4 \leq l\left(g_{T_{f l i p}}(v)\right) \leq l\left(g_{T}(v)\right)$, which permits to update the place of $v$ in a constant time.

Until : the "list of lists" is empty.

\subsubsection{Analysis}

Correctness : just before the $i+1^{s t}$ passage through the loop, a pair $\left(T_{1, i}, T_{2, i}\right)$ of tilings is stored. At the initialization, $\left(T_{1,0}, T_{2,0}\right)=\left(T, T^{\prime}\right)$, at each passage through the loop $\left(T_{1, i}, T_{2, i}\right)$ is replaced by a pair $\left(T_{1, i+1}, T_{2, i+1}\right)$ such that :

- $\operatorname{minflip}\left(T_{1, i+1}, T_{2, i+1}\right)=\operatorname{minflip}\left(T_{1, i}, T_{2, i}\right)-1$,

- either $T_{1, i+1}=T_{1, i}$ and $T_{2, i+1}$ is deduced from $T_{2, i}$ by a flip, or $T_{2, i+1}=$ $T_{2, i}$ and $T_{1, i+1}$ is deduced from $T_{1, i}$ by a flip.

The algorithm stops for the integer $i_{0}$ such that $T_{1, i_{0}}=T_{2, i_{0}}$.

We define a finite subsequence of $\left(T_{1,0}, T_{1,1}, \ldots T_{1, i_{0}}\right)$ constructed extracting different tilings : precisely $\left(T_{1,0}^{\prime}, T_{1,1}^{\prime}, \ldots T_{1, p}^{\prime}\right)$ is the sequence of tilings such that $T_{1,0}^{\prime}=T_{1,0}, T_{1, p}^{\prime}=T_{1, i_{0}}$ and for each integer $i$ such that $i<p, T_{1, i+1}^{\prime}$ equals the first element $T_{1, j}$ of the sequence $\left(T_{1,0}, T_{1,1}, \ldots T_{1, i_{0}}\right)$ such that $T_{1, j} \neq T_{1, i}^{\prime}$. We similarly define a subsequence $\left(T_{2,0}^{\prime}, T_{2,1}^{\prime}, \ldots T_{2, p^{\prime}}^{\prime}\right)$ of $\left(T_{2,0}, T_{2,1}, \ldots T_{2, i_{0}}\right)$.

By this way, the sequence $\left(T_{1,0}^{\prime}, T_{1,1}^{\prime}, \ldots T_{1, p}^{\prime}=T_{2, p^{\prime}}^{\prime}, T_{2, p^{\prime}-1}^{\prime}, \ldots T_{2,0}^{\prime}\right)$ is a shortest path of tilings from $T$ to $T^{\prime}$. The sequence of supports of flips necessary 
to pass from a tiling of this sequence to its successor is exactly the final list $L$, obtained at the end of the execution of the algorithm. This proves the correctness.

Time complexity : the characteristic values of a vertex $v$ can be deduced from those of its father in the spanning tree in $O(1)$ time units. Thus, the initialization can be done in $O(A(P))$ time units, where $A(P)$ denotes the area (i. e. the number of cells) of $P$.

Each passage through the loop costs $O(1)$ time units (for $m$ and $n$ been fixed). and reduces the distance between the current tiling and $T^{\prime}$ from $(m-$ $1)(n-1)$. Thus the second part of the algorithm costs at most $O\left(\Delta\left(T, T^{\prime}\right)\right)$ time units.

Thus the complete time cost is $O\left(A(P)+\Delta\left(T, T^{\prime}\right)\right)$, which is optimal since $O(A(P))$ time units are necessary to read the input and $O\left(\Delta\left(T, T^{\prime}\right)\right)$ time units are necessary to write the output.

\section{$5 \quad$ Structures of lattices and semi-lattices}

\subsection{Order relations on the set of tilings}

In this section, a tiling $T_{0}$ of $P$ is fixed and the tiling projection induced by $T_{0}$ is denoted by $g_{0}$.

Definition 8 Let $\left(T, T^{\prime}\right)$ be a pair of tilings of $P$. We say that $T \leq_{T_{0}} T^{\prime}$ if, for each vertex $v$ of $P, g_{0}(v)^{-1} g_{T}(v) \leq_{\text {decomp }} g_{0}(v)^{-1} g_{T^{\prime}}(v)$.

The relation defined by this way is obviously an order relation on the set $\Upsilon_{P}$ of tilings of $P$.The proposition below gives a geometrical interpretation of this order.

Proposition 7 Let $\left(T, T^{\prime}\right)$ be a pair of tilings of $P$. We have $T \leq_{T_{0}} T^{\prime}$ if and only if there exists a sequence $\left(T_{0}, T_{1}, \ldots, T_{p}\right)$ of tilings of $P$ such that : $T_{p}=T^{\prime}$, $p=\operatorname{minflip}\left(T_{0}, T^{\prime}\right)$, for each integer $i$ such that $0 \leq i<p ; T_{i}+1$ is deduced from $T_{i}$ by a local flip, and there exists an integer $i_{0}$ such that $0 \leq i_{0} \leq p$ and $T=T_{i_{0}}$.

Proof. $T \leq_{T_{0}} T^{\prime}$ if and only if we have the equality :

$$
\Delta\left(T_{0}, T^{\prime}\right)=\Delta\left(T_{0}, T\right)+\Delta\left(T, T^{\prime}\right)
$$

from the definition of the distance between tilings. Moreover, from the flip formula, the above equality is equivalent to :

$$
\operatorname{minflip}\left(T_{0}, T^{\prime}\right)=\operatorname{minflip}\left(T_{0}, T\right)+\operatorname{minflip}\left(T, T^{\prime}\right)
$$

which means that there exists a sequence of tilings as described in the proposition.

\subsection{The infimum property}

Proposition 8 Let $\left(T, T^{\prime}\right)$ be a pair of tilings of $P$. We define the function $g_{\text {inf }\left(T, T^{\prime}\right)}$ by, for each vertex $v$ of $P$,

$$
g_{\text {inf }\left(T, T^{\prime}\right)}(v)=g_{0}(v) i n f_{\text {decomp }}\left(g_{0}(v)^{-1} g_{T}(v), g_{0}(v)^{-1} g_{T^{\prime}}(v)\right)
$$

There exists a tiling $T$ " of $P$ such that $g_{T}=g_{\text {inf }\left(T, T^{\prime}\right)}$. 
The proof of the above proposition is based on the following lemma.

Lemma 4 Let $\left(w, w^{\prime}\right)$ be a pair of elements of ker $(s)$ such that in $f_{\text {decomp }}\left(w, w^{\prime}\right)=1_{G_{\text {cycles }}}$ and $\left(j, j^{\prime}\right)$ be a pair of integers. We state $u=$ $a^{-1} w b^{j} a b^{-j}$ and $u^{\prime}=a^{-1} w b^{j^{\prime}} a b^{-j^{\prime}}$ (notice that $u$ and $u^{\prime}$ both are elements of $\operatorname{ker}(s))$.

- If there exists an integer $j "$ such that $0<j "<n$ and $b^{j "} \leq$ cycles inf $f_{\text {cycles }}\left(w, w^{\prime}\right)$, then in $f_{\text {decomp }}\left(u, u^{\prime}\right)=a^{-1} b^{j "} a b^{-j^{\prime \prime}}$,

- otherwise, $i n f_{\text {decomp }}\left(u, u^{\prime}\right)=1_{G_{\text {cycles }}}$.

Proof. First assume that there exists an integer $j$ " such that $0<j "<n$ and $b^{j "} \leq_{\text {cycles }}$ in $f_{\text {cycles }}\left(w, w^{\prime}\right)$. Thus, we can state $w=b^{j \prime} w_{1}$ with the canonical expression of $w_{1}$ beginning by $a$ and $l\left(w_{1}\right) \geq 3($ since $l(w) \geq 4)$

If $l(w)=4$, then $w$ is element of $S_{\text {rect }}$, thus $l\left(w b^{j} a b^{-j}\right)=7$ and $\operatorname{init}\left(w b^{j} a b^{-j}\right)=\operatorname{init}(w)$. If $l(w) \geq 5$, we obviously have $l\left(w b^{j} a b^{-j}\right) \geq 2$ and $\operatorname{init}\left(w b^{j} a b^{-j}\right)=\operatorname{init}(w)$. Thus, in any case, we can state $w=b^{j "} a^{i} w_{1}$ with $0<i<m$ and either $w_{1}=1_{G_{c y c l e s}}$ or the canonical expression of $w_{1}$ begins by $b$.

In a similar way, we can state $w^{\prime} b^{j^{\prime}} a b^{-j^{\prime}}=b^{j^{\prime \prime}} a^{i^{\prime}} w_{1}^{\prime}$. Notice that $i \neq i^{\prime}$, from Lemma 1 . Thus, $u=\left(a^{-1} b^{j "} a b^{-j^{\prime \prime}}\right)\left(b^{j^{\prime \prime}} a^{-1+i} w_{1}\right)$ and $u^{\prime}=$ $\left(a^{-1} b^{j^{\prime \prime}} a b^{-j^{\prime \prime}}\right)\left(b^{j \prime} a^{-1+i^{\prime}} w_{1}^{\prime}\right)$, which gives the result, since $\operatorname{init}\left(b^{j^{\prime \prime}} a^{-1+i} w_{1}\right) \neq$ $\operatorname{init}\left(b^{j^{\prime \prime}} a^{-1+i^{\prime}} w_{1}\right)$.

Now we treat the second alternative of the lemma : if $w=a b^{j} a-1 b^{-j}$, then $u=1_{G_{\text {cycles }}}$, which obviously gives the result. The same argument can be used if $w^{\prime}=a b^{j^{\prime}} a-1 b^{-j^{\prime}}$ In any remaining case, one can remark as it has been done for the first alternative that $i n i t\left(w b^{j} a b^{-j}\right)=\operatorname{init}(w)$ and $\operatorname{init}\left(w^{\prime} b^{j} a b^{-j}\right)=\operatorname{init}\left(w^{\prime}\right)$. Thus $\operatorname{init}\left(w b^{j} a b^{-j}\right) \neq \operatorname{init}\left(w^{\prime} b^{j} a b^{-j}\right)$, which yields that $\operatorname{init}(u) \neq \operatorname{init}\left(u^{\prime}\right)$, which gives the result from Lemma 1.

Proof. (of Proposition 8) We prove this proposition, proving that $g_{i n f\left(T, T^{\prime}\right)}$ satisfies the hypothesis of proposition 2. The only non-trivial point is the verification that for each pair $\left(v, v^{\prime}\right)$ of neighbors vertices of $P$, $d\left(g_{\text {inf }\left(T, T^{\prime}\right)}(v), g_{\text {inf }\left(T, T^{\prime}\right)}\left(v^{\prime}\right)\right) \leq 3$.

We will prove it assuming, moreover, that $v^{\prime}=v a$ (which can be done without loss of generality, since the case when $v^{\prime}=v b$ can be treated in a symmetric way).

We need some notations : we state $g_{0}\left(v^{\prime}\right)=g_{0}(v) b^{j_{0}} a b^{-j_{0}}, g_{T}\left(v^{\prime}\right)=$ $g_{T}(v) b^{j_{T}} a b^{-j_{T}}$ and $g_{T^{\prime}}\left(v^{\prime}\right)=g_{T^{\prime}}(v) b^{j_{T^{\prime}}} a b^{-j_{T^{\prime}}}$.

We also state: $g_{i n f\left(T, T^{\prime}\right)}(v)^{-1} g_{0}(v)=w_{0}, g_{\text {inf }\left(T, T^{\prime}\right)}(v)^{-1} g_{T}(v)=$ $w_{T}, \quad g_{i n f\left(T, T^{\prime}\right)}(v)^{-1} g_{T^{\prime}}(v)=w_{T^{\prime}}$. With these notations, we have $i n f_{\text {decomp }}\left(w_{0}, w_{T}\right)=i n f_{\text {decomp }}\left(w_{0}, w_{T^{\prime}}\right)=i n f_{\text {decomp }}\left(w_{T}, w_{T^{\prime}}\right)=1_{G_{\text {cycles }}}$.

Afterwards, we state : $u_{0}=a^{-1} w_{0} b^{j_{0}} a b^{-j_{0}}, u_{T}=a^{-1} w_{T} b^{j_{T}} a b^{-j_{T}}$, $u_{T^{\prime}}=a^{-1} w_{T^{\prime}} b^{j_{T^{\prime}}} a b^{-j_{T^{\prime}}}$.

A) If there exists an integer $j "$ such that $0<j "<n$ and $b^{j "} \leq_{\text {cycles }}$ in $f_{\text {cycles }}\left(w_{0}, w_{T}, w_{T^{\prime}}\right)$, then, from the previous lemma, we have :

$$
i n f_{\text {decomp }}\left(u_{0}, u_{T}\right)=i n f_{\text {decomp }}\left(u_{0}, u_{T^{\prime}}\right)=i n f_{\text {decomp }}\left(u_{T}, u_{T^{\prime}}\right)=a^{-1} b^{j^{\prime \prime}} a b^{-j^{\prime \prime}}
$$


Thus, if we state $t=a^{-1} b^{j "} a b^{-j "}$, we have :

$\inf f_{\text {decomp }}\left(t^{-1} u_{0}, t^{-1} u_{T}\right)=i n f_{\text {decomp }}\left(t^{-1} u_{0}, t^{-1} u_{T^{\prime}}\right)=i n f_{\text {decomp }}\left(t^{-1} u_{T}, t^{-1} u_{T^{\prime}}\right)=1_{G_{\text {cycles }}}$

which means that $g_{i n f\left(T, T^{\prime}\right)}(v a)=g_{i n f\left(T, T^{\prime}\right)}(v) b^{j^{\prime \prime}} a b^{-j^{\prime \prime}}$ and gives the result.

B) If there exists an integer $j "$ such that $0<j "<n$ and $b^{j "} \leq_{\text {cycles }}$ $i n f_{\text {cycles }}\left(w_{0}, w_{T}\right)$ (which yields that in $f_{\text {decomp }}\left(u_{0}, u_{T}\right)=a^{-1} b^{j} a b^{-j "}=t$ ), and inf $f_{\text {decomp }}\left(u_{0}, u_{T^{\prime}}\right)=i n f_{\text {decomp }}\left(u_{T}, u_{T^{\prime}}\right)=1_{G_{\text {cycles }}}$, then

$\inf f_{\text {decomp }}\left(t^{-1} u_{0}, t^{-1} u_{T}\right)=i n f_{\text {decomp }}\left(t^{-1} u_{0}, t^{-1} u_{T^{\prime}}\right)=i n f_{\text {decomp }}\left(t^{-1} u_{T}, t^{-1} u_{T^{\prime}}\right)=1_{G_{\text {cycles }}}$ which means that $g_{i n f\left(T, T^{\prime}\right)}(v a)=g_{i n f\left(T, T^{\prime}\right)}(v) b^{j^{\prime \prime}} a b^{-j^{\prime \prime}}$ and gives the result.

C) If $i n f_{\text {decomp }}\left(u_{0}, u_{T}\right)=i n f_{\text {decomp }}\left(u_{0}, u_{T^{\prime}}\right)=i n f_{\text {decomp }}\left(u_{T}, u_{T^{\prime}}\right)=$ $1_{G_{c y c l e s}}$, then we have $g_{i n f\left(T, T^{\prime}\right)}(v a)=g_{i n f\left(T, T^{\prime}\right)}(v) a$, which gives the result.

We have treated all the cases, (up to symmetry), from the previous lemma.

Corollary 2 For each tiling $T_{0}$ of $P$, the order relation $\left(\Upsilon_{P}, \leq_{T_{0}}\right)$ is an inferior semi-lattice.

Proof. obvious.

Proposition 9 For each pair $\left(T_{0}, T_{0}^{\prime}\right)$ of tilings of $P$, we define the set $\Upsilon_{T_{0}, T_{0}^{\prime}}$ formed from tilings $T$ such that $T_{0} \leq_{T_{0}} T \leq_{T_{0}} T_{0}^{\prime}$.

The order relation $\left(\Upsilon_{T_{0}, T_{0}^{\prime}}, \leq_{T_{0}}\right)$ is a distributive lattice.

Proof. We have seen that $\Upsilon_{T_{0}, T_{0}^{\prime}}$ has the minimum property. Notice that $T_{0} \leq T_{0}$ $T \leq_{T_{0}} T_{0}^{\prime}$ if and only if $T_{0}^{\prime} \leq_{T_{0}^{\prime}} T \leq_{T_{0}^{\prime}} T_{0}$. Thus $\Upsilon_{T_{0}, T_{0}^{\prime}}$ has the maximum property, since $\Upsilon_{T_{0}^{\prime}, T_{0}}$ has the infimum property. We have proven that $\Upsilon_{T_{0}, T_{0}^{\prime}}$ has a lattice structure.

For each vertex $v$ of $P$, we state $g_{T_{0}^{\prime}}(v)=g_{T_{0}}(v) \Pi_{i=1}^{p(v)} t_{i}(v)$, where $\Pi_{i=1}^{p(v)} t_{i}(v)$ is the decomposition of $g_{T_{0}}(v)^{-1} g_{T_{0}^{\prime}}(v)$. Let $T$ be a tiling of $\Upsilon_{T_{0}, T_{0}^{\prime}}$. There exists a unique integer $q_{T}(v)$ such that $0 \leq q(v) \leq p(v)$ and $g_{T}(v)=g_{T_{0}}(v) \Pi_{i=1}^{q_{T}}(v) t_{i}(v)$. Thus, one can define the injective mapping $Q$ from $\Upsilon_{T_{0}, T_{0}^{\prime}}$ to $Z^{V}$ (where $V$ denotes the set of vertices of $P$ ) such that $Q(T)$ is the vector formed from values $q_{T}(v)$.

By definition of the order on $\Upsilon_{T_{0}, T_{0}^{\prime}}$, the mapping $Q$ is a lattice morphism (i. e. $Q\left(\inf \left(T, T^{\prime}\right)\right)=\inf \left(Q(T), Q\left(T^{\prime}\right)\right)$ and $\left.Q\left(\sup \left(T, T^{\prime}\right)\right)=\sup \left(Q(T), Q\left(T^{\prime}\right)\right)\right)$, which yields that $\Upsilon_{T_{0}, T_{0}^{\prime}}$ is isomorphic to a sublattice of $Z^{V}$ and, consequently, is a distributive lattice.

\section{Tilings with leaning dominoes and triangles}

From a similar method, we will see that similar results and algorithms can be obtained working with sets of prototiles of the triangular lattice. In this section 
we limit ourselves to present the general framework and the main tools used, and do not give proofs, since most of they are very similar to those of previous sections, about tilings with bars.

The triangular lattice $\Gamma$ induces three unit moves in the plane : rightwards, denoted by $a$, and $b$ and $c$, such that angle $(a, b)=\operatorname{angle}(b, c)=\operatorname{angle}(c, a)=$ $2 \Pi / 3$. Let $v$ be a vertex of $\Gamma$ and $u$ denote an element of $\left\{a, b, c, a^{-1}, b^{-1}, c^{-1}\right\}$. The element of $\Gamma$ which is reached from $v$ with a $u$-move is denoted by $v u$.

We now study the set of prototiles $S=\left\{l d_{1}, l d_{2}, l d_{3}, l d_{4}, l d_{5}, l d_{6}, t r_{1}, t r_{2}\right\}$ (previously studied in [5]), where $l d_{i}$ (respectively $t r_{i}$ ) denotes a parallelogram (respectively an equilateral triangle) formed from four cells of $\Gamma$. Each prototile $l d_{i}$ is called a leaning domino.

A set of contour words of $S$ is $R=$ $\left\{a^{2} b a^{-2} b^{-1}, a^{2} c a^{-2} c^{-1}, b^{2} a b^{-2} a^{-1}, b^{2} c b^{-2} c^{-1}, c^{2} a c^{-2} a^{-1}\right.$,

$\left.c^{2} b c^{-2} b^{-1}, a^{2} b^{2} c^{2}, a^{2} c^{2} b^{2}\right\}$,thus the tiling group $G_{t i l e}$ is the group generated by $\{a, b, c\}$ whose set of relators is $R$ (i. e. the quotient group $F_{a, b, c} / N_{R}$ )
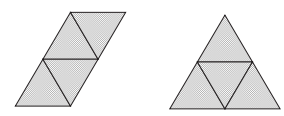

Figure 5: A leaning domino and a triangular prototile. Counterclockwise, starting from the lower left corners, the contour words respectively are $a c^{-2} a^{-1} c^{2}$ and $a^{2} b^{2} c^{2}$.

\subsection{Quotient groups}

\subsubsection{The tricolored group}

Since $G_{t i l e}$ is complex, we use quotients of it. The main quotient group used is $\left\langle a, b, c \mid a^{2}, b^{2}, c^{2}\right\rangle$. This group is isomorphic of the free product of three groups, each of them with only two elements. If we identify opposite arcs with the same label, the induced Cayley graph is a tree.

Each element $w$ of $G_{\text {tricolored }}$ has a canonical expression : $w$ can be written in a unique way as $w=\prod_{i=1}^{p} x_{i}$ with, for each integer $i, x_{i} \in\{a, b, c\}$ and, for $i<p, x_{i} \neq x_{i+1}$. This permits to define the initial (respectively final part) of $w$ (the word formed by the two first (respectively last) letters of the canonical expression of $w)$, the length $l(w)$ of $w$ by $: l(w)=p$ and the distance $d\left(w^{\prime}, w^{\prime \prime}\right)$ between elements of $G_{\text {tricolored }}$ by $d\left(w^{\prime}, w^{\prime \prime}\right)=l\left(w^{\prime-1} w^{\prime \prime}\right)$. One can also canonically define an order relation (denoted by $\leq_{\text {tricolored }}$ ) on $G_{\text {tricolored }}$. This relation obviously has the infimum property.

Let $P$ be a fixed polygon formed from cells of the triangular lattice and $v_{0}$ be a fixed vertex of the boundary of $P$. As in Section 3, for each tiling $T$ of $P$, one can define a tiling projection $g_{T}$, which associates an element of $G_{\text {tricolored }}$ to each vertex $v$ of the polygon. Such a tiling projection encodes the tiling $T$.

\subsubsection{The cell group and the tetrahedron group}

Another quotient group of $G_{t i l e}$ is $G_{c e l l}=\langle a, b, c| a b c, a c b>$ (i. e. the set of relators is a set of contour words of cells). This group is isomorphic to $Z^{2}$ and 


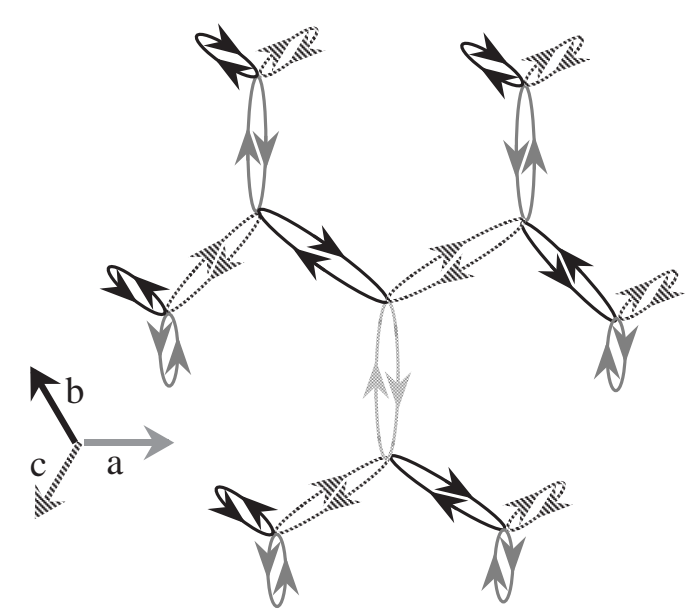

Figure 6: The Cayley graph of $G_{\text {tricolored }}$

the induced Cayley graph is the planar triangular grid.

The third quotient group is $G_{\text {tetrahedron }}=<a, b, c \mid a^{2}, b^{2}, c^{2}, a b c, a c b>$. This group has four elements and the induced Cayley graph is a tetrahedron. Since the exists a canonical morphism from $G_{\text {cell }}$ (which can be seen as the planar triangular grid) to $G_{\text {tetrahedron, }}$, we can define (after an origin vertex $v_{0}$ has been fixed), for each vertex $v$ of the grid, the element $\operatorname{cong}_{\text {tetrahedron }}(v)$ of $G_{\text {tetrahedron }}$.

Given a tiling $T$ of a polygon $P$ with a fixed vertex $v_{0}$ of its boundary, one can define a tiling projection $g_{T}$ from vertices of $P$ to $G_{\text {tricolored }}$. We have, in a similar way as in Section 3, the proposition below.

Proposition 10 Let $g$ be a function from the set of vertices of $P$ to $G_{\text {tricolored }}$. There exists a tiling $T$ of $P$ such that $g=g_{T}$ if and only if the following constraints are satisfied:

- $g\left(v_{0}\right)=1_{G_{\text {tricolored }}}$,

- for each vertex vertex $v$ of $P, s(g(v))=\operatorname{cong}_{\text {tetrahedron }}(v)$,

- for each pair $\left(v, v^{\prime}\right)$ of neighbors vertices of $P, d\left(g(v), g\left(v^{\prime}\right)\right) \leq 3$, and if, moreover, the line segment $\left[v, v^{\prime}\right]$ is included on the boundary of $P$, then $d\left(g(v), g\left(v^{\prime}\right)\right)=1$.

Proof. (sketch) The direct part of the proposition is very easy. Conversely, assume that $g$ satisfies the constraint above and let $[v, v a]$ be a line segment included in $P$. We necessarily have $g(v)^{-1} g(v a) \in\{a, b c, c b, c a c, b a b\}$ (we have a similar fact for line segments $[v, v b]$ and $[v, v c])$.

Moreover, we have a large information about the values of $g\left(v b^{-1}\right)$ and $g\left(v c^{-1}\right)$ : precisely, if $g(v)^{-1} g(v a)=b a b$, then $g(v)^{-1} g\left(v c^{-1}\right)=b a$ and $g(v)^{-1} g\left(v b^{-1}\right)=b$; if $g(v)^{-1} g(v a)=b c$, then $g(v)^{-1} g\left(v b^{-1}\right)=b$ and $g(v)^{-1} g\left(v b^{-1}\right) \in\{c, b a, b c b\}$ (we also have a lot of symmetric equalities).

The above equalities imply that the set of edges $\left[v, v^{\prime}\right]$ such that $d\left(g(v), g\left(v^{\prime}\right)\right)=1$ draw a tiling $T$ of $P$ : precisely, for each cell $C$ of $P$, the 
set of cells $C^{\prime}$ such that there exists a path starting in $C$ and finishing in $C^{\prime}$ which cuts no edge $\left[v, v^{\prime}\right]$ such that $d\left(g(v), g\left(v^{\prime}\right)\right)=1$, form a tile. The set of those tiles form a tiling $T$ of $P$ and we obviously have $g=g_{T}$.

As a corollary, we obtain that, for each pair $\left(T, T^{\prime}\right)$ of tilings of $P$ and for each vertex $v$ of $P, g_{T^{\prime}}(v)^{-1} g_{T}(v)$ is element of the kernel of the canonical morphism from $G_{\text {tricolored }}$ to $G_{\text {tetrahedron }}$. For the following, this kernel is denoted by $N_{\text {cell }}^{\prime}$.

\subsubsection{Structures induced by $N_{\text {cell }}^{\prime}$}

We state $S_{\text {triangle }}=\{a b c, a c b, b a c, b c a, c a b, c b a\}$ (i. e. the set of possibles contour words of triangular cells). As in Section 3, one can prove that each element $w$ of $N_{\text {cell }}^{\prime}$ can be written in a unique way as $w=\Pi_{i=1}^{p^{\prime}} x_{i}$ with, for each integer $i, x_{i} \in S_{\text {triangle }}$ and, for $i<p, x_{i} \neq x_{i+1}$.

Moreover, the canonical expression of $w$ finishes by $a b$ (respectively $a c, b a$, $b c, c a, c b$ ) if and only if $x_{p^{\prime}}=c a b$ (respectively $b a c, c b a, a b c, b c a, a c b$ ).

Thus, as in Section 3, one can define the order relation $\leq_{\text {decomp }}$ on $N_{\text {cell }}^{\prime}$, the decomposition number num $(w)$ of $w$ by num $(w)=p^{\prime}$, and, afterwards, the distance $\Delta\left(T, T^{\prime}\right)$ between two tilings of a same polygon $P$.

\subsection{Local flips}

We have to kind of local flips (see figure 7 ) : a lozenge $L_{0}$, formed from height triangular cells of $P$ admits three tilings. Two of those tilings are formed from two leaning dominoes and the third one is formed from two triangles. The replacement of a tiling of $L_{0}$ formed from parallelograms by a tiling formed from triangles (or the inverse) is our first kind of local flip (the lozenge flips).

An isoceles trapeze $T r_{0}$ formed from height triangular cells of $P$ admits two tilings, each of them formed from a parallelogram and a triangle. The replacement of one of these two tilings by the other one is our second kind of local rotations (the trapeze flips).

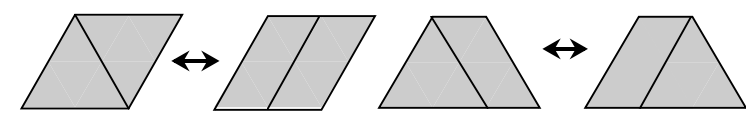

Figure 7: Flips for leaning dominoes and triangles

Let $T$ be a tiling and $T_{f l i p}$ be a tiling deduced by a flip of support Sup. (which is either a lozenge or a trapeze). Let $v_{\text {Sup }}$ denote the only vertex which is in the interior part of Sup. For each vertex $v$ of $P$ such that $v \neq v_{\text {Sup }}$, we have $g_{T}(v)=g_{T_{f l i p}}(v)$ and $g_{T}\left(v_{\text {Sup }}\right)^{-1} g_{T_{\text {flip }}}\left(v_{\text {Sup }}\right)$ is element of $S_{\text {triangle }}$.

\subsubsection{Maximal vertex}

A maximal vertex for a pair $\left(T, T^{\prime}\right)$ of tilings can be defined exactly as in section 3 , but the use of maximal vertices is a little different.

Let $v_{1}$ be a maximal vertex for a pair $\left(T, T^{\prime}\right)$ of distinct tilings. One can assume without loss of generality that $l\left(g_{T^{\prime}}\left(v_{1}\right)\right) \leq l\left(g_{T}\left(v_{1}\right)\right)$. 
From the tree structure of $G_{\text {tricolored }}$, one easily proves that $l\left(g_{T}\left(v_{1}\right)\right) \geq 2$. Moreover, if the final part of $g_{T}\left(v_{1}\right)$ is $a b$, then, since $l\left(g_{T^{\prime}}\left(v_{1}\right)\right) \leq l\left(g_{T}\left(v_{1}\right)\right)$, the last factor of the decomposition of $g_{T^{\prime}}\left(v_{1}\right)^{-1} g_{T}\left(v_{1}\right)$ is necessarily $c a b$.

Proposition 11 Consider the line segment $\left[v_{1} b^{-1}, v_{1} b\right]$. This line segment is the common side of two tiles of $T$. Moreover, each leaning domino of $T$ a large side of which is $\left[v_{1} b^{-1}, v_{1} b\right]$ admits $b^{2} a^{-1} b^{-2} a$ as contour word.

Proof. (sketch) We first claim that the line segment $\left[v_{1}, v_{1} c\right]$ necessarily cuts a tile of $T$ : otherwise, we have $l\left(g_{T}\left(v_{1} c\right)\right)=l\left(g_{T}\left(v_{1}\right)\right)+1$.

On the other hand, since the final part of $g_{T^{\prime}}\left(v_{1}\right)^{-1} g_{T}\left(v_{1}\right)$ is $a b$, we have

$$
d\left(g_{T^{\prime}}\left(v_{1}\right), g_{T}\left(v_{1} c\right)\right)=d\left(g_{T^{\prime}}\left(v_{1}\right), g_{T}\left(v_{1}\right)\right)+1 \geq 3+1=4
$$

These inequalities prove that $g_{T}\left(v_{1} c\right) \neq g_{T^{\prime}}\left(v_{1} c\right)$, since $d\left(g_{T^{\prime}}\left(v_{1}\right), g_{T^{\prime}}\left(v_{1} c\right)\right) \leq$ 3. Thus $v_{1} c$ contradicts the maximality of $v_{1}$.

The same argument can also be used for $v_{1} a, v_{1} c^{-1}$ and $v_{1} a^{-1}$. This gives the first part of the proposition.

If we assume that a leaning domino, whose sides issued from $v_{1} b^{-1}$ are $\left[v_{1} b^{-1}, v_{1} b\right]$ and $\left[v_{1} b^{-1}, v_{1} b^{-1} c\right]$, is element of $T$, then one proves as above that $l\left(g_{T}\left(v_{1} c\right)\right)=l\left(g_{T}\left(v_{1}\right)\right)+1$ and $g_{T}\left(v_{1} c\right) \neq g_{T^{\prime}}\left(v_{1} c\right)$, which contradicts the maximality of $v_{1}$. This gives the second part of the proposition.

From the above proposition, it follows that a flip can be done around such an extremal vertex $v_{1}$. This flip decreases the distance between $T$ and $T^{\prime}$. Thus, by a similar study as in Section 3, we obtain :

- a flip formula : $\operatorname{minflip}\left(T, T^{\prime}\right)==\sum_{v \in P} n u m\left(g_{T^{\prime}}(v)^{-1} g_{T}(v)\right)$,

- an algorithm which, given a pair of tilings, produces a sequence of minimal length of necessary flips to transform the first tiling into the second one.

\subsection{Lattice structures}

For each tiling $T_{0}$, we can define, as in Section 5 , an order relation $\leq_{T_{0}}$, which can be geometrically interpreted by : for each pair $\left(T, T^{\prime}\right)$ of tilings of $P, T \leq_{T_{0}} T^{\prime}$ if and only if there exists a sequence $\left(T_{0}, T_{1}, \ldots, T_{p}\right)$ of tilings of $P$ such that $: T_{p}=T^{\prime}, p=\operatorname{minflip}\left(T_{0}, T^{\prime}\right)$, for each integer $i$ such that $0 \leq i<p ; T_{i}+1$ is deduced from $T_{i}$ by a local flip, and there exists an integer $i_{0}$ such that $0 \leq i_{0} \leq p$ and $T=T_{i_{0}}$.

Lemma 5 Let $\left(w, w^{\prime}\right)$ be a pair of elements of $N_{c e l l}^{\prime}$ such that inf $f_{\text {decomp }}\left(w, w^{\prime}\right)=1_{G_{\text {tricolored }}}$ and $\left(s, s^{\prime}\right)$ be a pair of elements of $\{a, b c, c b, c a c, b a b\}$. We state $u=$ aws and $u^{\prime}=a w s^{\prime}$ (notice that $u$ and $u^{\prime}$ both are elements of $N_{\text {cell }}^{\prime}$ ).

- If $b \leq_{\text {tricolored }}$ in $f_{\text {tricolored }}\left(w, w^{\prime}\right)$, then in $f_{\text {decomp }}\left(u, u^{\prime}\right)=a b c$,

- If $c \leq_{\text {tricolored }}$ in $f_{\text {tricolored }}\left(w, w^{\prime}\right)$, then in $f_{\text {decomp }}\left(u, u^{\prime}\right)=a c b$,

- otherwise, $\inf f_{\text {decomp }}\left(u, u^{\prime}\right)=1_{G_{\text {tricolored }}}$. 
From this lemma, as in Section 5, one deduces that the order $\leq_{\text {tricolored }}$ induces a structure of inferior semi-lattice on the set of tilings of $P$, with $T_{0}$ as minimum element, and a structure of distributive lattice for each interval of tilings.

\section{Tilings with calissons}

The same method can easily be applied to the set of prototiles $S=$ $\left\{\mathrm{cal}_{1}, \mathrm{cal}_{2}, \mathrm{cal}_{3}\right\}$ (the calissons, studied in [9]), each element of $S$ being formed from two cells of $\Gamma$ with a common edge. A set of contour words is $\left\{a b a^{-1} b^{-1}, a c a^{-1} c^{-1}, b c b^{-1} c^{-1}\right\}$.

The main quotient group used is $G_{\text {line }}=\left\langle a, b \mid a b^{-1}, a c^{-1}\right\rangle$. Each element $w$ of $G_{\text {line }}$ has a canonical expression : there exists a unique relative integer such that $w=a^{p}$. This permits to define the length $l(w)$ of $w$ by $l(w)=|p|$.

Given a tiling $T$ of a polygon $P$, one can define a tiling projection $g_{T}$ from vertices of $P$ to $G_{\text {line }}$. One can prove that, for each vertex $v$ of $P, g_{T^{\prime}}(v)^{-1} g_{T}(v)$ is element of the normal group $N$ "cell of $G_{\text {line }}$ generated by $\{a b c, a c b\}=\left\{a^{3}\right\}$. Thus, each element $w$ of $N$ "cell can be written in a unique way as $w=a^{3 p}$. This permits to define the decomposition number num $(w)$ of $w$ by num $(w)=$ $\left|p^{\prime}\right|(=l(w) / 3)$, and, afterwards, the distance $\Delta\left(T, T^{\prime}\right)$ between two tilings of a same polygon $P$.

The local flips are induced by the two possible tilings or a hexagon formed from six cells of $\Gamma$ (see figure 8 ). In this case, the end of the study is very simple, since $G_{\text {line }}$ is isomorphic to $Z$. we obtain :

- a flip formula : $\operatorname{minflip}\left(T, T^{\prime}\right)=\sum_{v \in P}\left|l\left(g_{T^{\prime}}\right)(v)-l\left(g_{T}(v)\right)\right| / 3$,

- an algorithm which, given a pair of tilings, produces a sequence of minimal length of necessary flips to transform the first tiling into the second one,

- a structure of distributive lattice in the set of tilings (the addition of a fictive maximum is not needed because of the structure of line of the quotient group).

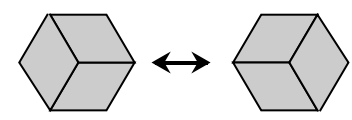

Figure 8: Flips for calissons

As for the dominoes, results about calissons were previously obtained using elementary methods $([6],[8])$, but here, we explain them with a general framework.

\section{References}

[1] J. H. Conway, J. C. Lagarias, Tiling with Polyominoes and Combinatorial Group Theory, Journal of Combinatorial Theory A 53 (1990) p. 183-208. 
[2] C. Kenyon, R. Kenyon, Tiling a polygon with rectangles, proceedings of the $33^{r d}$ IEEE conference on Foundations of Computer Science (FOCS) (1992) p. 610-619.

[3] J. C. Lagarias, D. S. Romano, A Polyomino Tiling of Thurston and its Configurational Entropy, Journal of Combinatorial Theory A 63 (1993) p. 338-358.

[4] J. G. Propp, Lattice structure for orientations of graphs, preprint.

[5] E. Rémila, Tiling groups : new applications in the triangular lattice, Discrete and Computational Geometry 20 (1998), p. 189-204.

[6] E. Rémila, On the lattice structure of the set of tilings of a simply connected figure with dominoes, proceedings of the $3^{\text {rd }}$ International Conference on Orders, Algorithms and Applications (ORDAL) LNCS Springer (to appear) 12 pages.

[7] E. Rémila, An algebraic method to compute a shortest path of local flips between two tilings, proceedings of the $11^{\text {th }}$ annual ACM-SIAM Symposium On Discrete Algorithms (SODA) SIAM eds, Philadelphia, (2000) p. 646653.

[8] N. C. Saldanha, C. Tomei, M. A. Casarin Jr, D. Romualdo, Spaces of domino tilings, Discrete Computational Geometry 14 (1995), p . 207-233.

[9] W. P. Thurston, Conway's tiling groups, American Mathematical Monthly, (1990) p. $757-773$. 